\title{
CHINESE WALL SECURITY POLICY MODELS
}

\section{Information Flows and Confining Trojan Horses}

\author{
Tsau Young ('T. Y.')Lin \\ Department of Computer Science \\ San Jose State University \\ San Jose, California 95192 \\ tylin@cs.sjsu.edu
}

\begin{abstract}
In 1989, Brewer and Nash (BN) presented a fascinating idea, called Chinese wall security policy model, for commercial security. Their idea was based on the analysis of the notion, Conflict of Interest binary Relation (CIR). Unfortunately, their formalization did not fully catch the appropriate properties of CIR. In this paper, we present a theory based on granulation that has captured the essence of BN's intuitive idea. The results are more than the Chinese wall models: Malicious Trojan horses in certain DAC Model (discretionary access control) can be controlled or confined.
\end{abstract}

Keywords: Simple Chinese Wall Security policy, Agressive(Strong) Chinese Wall Security policy, binary relation, conflict of interests, equivlaence relation.

\section{Introduction}

Recent developments, such as eCommerce and homeland security, have prompted us to re-visit the idea of Chinese Wall Security Policy (CWSP) model. "The Chinese wall policy combines commercial discretion with legally enforceable mandatory controls ..., perhaps, as significant to the financial world as Bell-LaPadula's policies are to the military." This is an assertion made by Brewer and Nash; see the abstract of [2]. We believe it is still valid.

In 1989, Brewer and Nash(BN) proposed a very intriguing commercial security model - called Chinese Wall Security Policy(CWSP) Model [2]. Intuitively, the essential idea was to build a family of impenetrable walls, called Chinese walls, among the dataset of competing companies. 
No data that are in conflict can be stored in the same side of Chinese walls. BN's analysis of the security problem were fascinating and is still valid; the only unfortunate part is their mathematical model has not fully captured their analysis. It was based on

- The incorrect assumption that corporate data can be partitioned (decomposed) into mutually disjoin conflict of interest classes (CIRclasses); such a disjoint collection is called a partition in mathematics; see e.g., [3].

- Unfortunately, CIR-classes seldom disjoints; they do overlap, and hence Brewer and Nash theory does not held well.

In the same year at 1989 Aerospace Computer Security Application Conference, we reported BN's errors, and presented a modified model, called an aggressive Chinese Wall Security Policy model (ACWSP) [14]. In that paper, we did not bring out the essential strength of ACWSP model. A relatively inactive decade has passed; only few papers, which are, however, still based on the same erroneous assumptions, appeared. By refining the idea of ACWSP, in this paper, we have successfully captured the intuitive intention of BN "theory."

The theory that we are presenting is based on the development of a novel computing methodology. We observed that

- The collection of CIR-classes form a binary granulation, even though not a partition.

Intuitively a binary granulation is a collection of subsets, whose "core" or "centers" form a partition; see the main text below. In terms of binary granulation, we recapture the spirit of Chinese wall security policy (CWSP) model. The results are more than that; with mild assumptions, we can actually confine malicious Trojan horses on DAC model (Discretionary Access Control). Sensitive information will not flow into "enemy" hands, only flow among "friends."

\section{An Analysis of Conflicts(CIR)}

Before we launch on the new theory, we recall some analysis from [14]: Conflict of Interests is a binary Relation (CIR) among corporations; it can be used to regulate the information flows among corporations.

\subsection{Is CIR an equivalence Relation?}

In $\mathrm{BN}$-theory, $\mathrm{CIR}$ were assumed to be an equivalence relation erroneously, in other words, $O$ were partitioned (decomposed) into mutually disjoint CIR-classes (conflict of interest classes) However, "conflict 
of interests," in normal daily usage, can not be transitive, at least not universally transitive.

Let us recall the example from [14]: The following two statements, at least, during the cold war period were valid.

USA was in cold war with USSR, and

USSR was in cold war with UK.

Could we conclude from these two assertions that

USA was in cold war with USSR.

This is rather absurd. Of course, in cold war with, is only an example, nevertheless, it does clearly indicate that CIR can not, in general, be transitive. So an equivalence relation, which is reflexive, symmetric and transitive, is not a proper mathematical model for CIR.

\subsection{A Set of Axioms for Secure CIR}

In this section, we re-visit the Aggressive Chinese Wall Security Policy(ACWSP) Model [14]. We recall (with some modifications) the axioms for the binary relation, $C I R \subseteq O \times O$ :

CIR-1: CIR is symmetric.

CIR-2: CIR is anti-reflexive.

CIR-3: CIR is anti-transitive.

Some observations may be helpful: CIR-2 is necessary, since each company cannot conflict to itself. CIR- 1 is always valid: If company $A$ is in conflicts with B, B is certainly in conflicts with A. CIR-3 is imposed, because it implicitly required in BN's notion of Chinese walls (Section 3.1).

To stress its importancy, a new property will be explicitly listed as an "axiom", though it is implied by the others: Let $E_{C I R}$ be the induced equivalence relation of CIR(See Section 3).

CIR-4: The granulation of CIR and partition of $E_{C I R}$ are compatible, in the sense that each CIR-class (or in geometric term, CIRneighborhood) is a union of $E_{C I R}$-equivalence classes. (See Proposition 2 of Section 3)

Intuitively, this is significantl; previously we have placed Chinese walls on the "boundaries" of CIR-classes in[14]. But each of these boundaries is "one-sided;" its complement is not an union of CIR-classes. But CIR-4 implies that that such boundaries are "two-sided;" Because the "interior and exterior" are both unions of $E_{C I R^{-}}$equivalence classes. 
CIR-5 (IAR): If we interpret CIR as "is an adversary of"-relation, then the complement is "is an ally of"-relation (IAR). IAR is an equivalence relation, by Corollary 4 of Section 4 .

Theorem 2.2. If $\mathrm{R}$ is a symmetric and anti-reflexive and anti-transitive binary relation, its complement $\mathrm{R}^{\prime}$ is an equivalence relation. In particular, IAR, as the the complement of CIR, is an equivalence relation.

Proof: Let $[X]_{I A R}$ be the IAR-equivalence class containing $X$. Then, we note that $[X]_{I A R}=[Y]_{I A R} \forall Y \in[X]_{I A R}$. Hence their complements are equal, so we have

Corollary $C I R_{X}=C I R_{Y} \forall Y \in[X]_{I A R}$. In other words, the information of any object in $[X]_{I A R}$ can flow freely among themselves

\section{Chinese Wall Security Policies}

$\mathrm{BN}$ proposed Chinese wall security policy to capture certain requirments in UK's financial sector. A decade later, we beleive their anlaysis is still useful and valid (but not their mathematical model). In this section, we will re-visit their requirements, namely, Chinese Wall Security Policy (CWSP).

\subsection{Simple Chinese Wall Security Policy}

We will continue on BN's notations: $O$ is the set of all objects (corporate data), $X, Y$, and $Z$ are objects in $O$.

1. BN-Version : "people are only allowed access to information which is not held to conflict with any other information that they already possess." See [2], Section "Simple Security", p. 207.

We will rephrese it mathemtically:

2. SCWSP Simple Chinese Wall Security Policy means

There is no DIF from $X$ to $Y$, if and only if $(X, Y) \in \mathrm{CIR}$,

where DIF (direct information flows) means the agent $y$ (who handles $Y$ ) read the data in $Y$ (and may make a copy in $Y$ ).

In terms of DAC(Descretionary Acess Model) langauge, we should say $x$ grants $y$ a read-access right; and $y$ read the data and make a copy in $Y$ (making copy is permissible, even in strcit DAC model; see [23])

Please note the necessary-and-sufficient-condition type of statements. BN's rules allow an agent to read any two sets of data as long as they are not CIR. So we havw the dual of SCWSP: 
3. Dual-SCWSP Dual-SCWSP is equivalent to CWSP, but opposite View:

There is DIF from $X$ to $Y$, if and only if $(X, Y) \notin C I R$.

\subsection{Agressive Chinese Wall Security Policy (ACWSP)}

First, let us state a convention, some definitions and comments:

Convention : The symbol, CIR, always denotes a binary relation that satisfies the axioms.

Definition CIF (composite information flow) from $X$ to $Y$ is a sequence of DIFs (direct information flows), which starts from $X$ and end at $Y$ :

$$
X=X_{0} \longrightarrow X_{1} \longrightarrow \ldots \rightarrow X_{n}=Y,
$$

where each " $\longrightarrow$ ” is a DIF.

Caution : We should stress here that simple security policy only regulate DIF's. Note that even if we are assured that there is no DIF from $X$ to $Y$, it is conceivable some clever CIFs may send information, say from $X$, via $Z$, to $Y$. In other words, two innocent DIFs from $X \longrightarrow Z$ and $Z \longrightarrow Y$ could compose into a malicious $\mathrm{CIF}, X \longrightarrow Z \longrightarrow Y$.

Such CIFs have been identified as Trojan horses. Confining the malicious Trojan horses is actually the essence of the main contribution of ACWSP-model.

\section{ACWSP Agressive (Strong) Chinese Wall Security policy.}

There is no CIF from $X$ to $Y$, if and only if $(X, Y) \in$ CIR.

Chinese Wall Theorem. Under the assumption that CIR meets the axioms (anti-reflexive, symmetric, and anti-transitive),

\section{ACWSP $\Longleftrightarrow$ SCWSP}

Proof: First, let us note the following FACTs from Section ??

FACT 1. CIR and IAR("is ally with") are complement to each other.

FACT 2. IAR is an equivalence relation. 
We need some notations. Let $X$ be an object (a company data) of $O$. Let

$C I R_{X}(=\{Y \mid(X, Y) \in \mathrm{CIR}\}$ be the CIR-class of $X$ and

$[X]_{I A R}(=\{Y \mid(X, Y) \in$ IAR $\})$, or simply $[X]$, IAR-equivalence class of $X$

Let $X$ be an object (a company data) of $O$. Let

$$
\begin{aligned}
& \operatorname{CIR}_{X}(=\{Y \mid(X, Y) \in \mathrm{CIR}\} \text { be the CIR-class of } X \text { and } \\
& {[X]_{\text {IAR }}(=\{Y \mid(X, Y) \in \mathrm{IAR}\}) \text {, or simply }[X], \text { IAR-equivalence }} \\
& \text { class of } X
\end{aligned}
$$

To prove this theorem, we need to establish the following Agressive(strong)

Chinese wall security policy for the given Simple CWSP:

There is no CIF from $X$ to $Y \Longleftarrow(X, Y) \in \mathrm{CIR}$.

We will use indirect proof, so by assuming to the contrary, we have two conditions:

1. There is a sequence of DIFs (direct information flows)

$$
X=X_{0} \longrightarrow X_{1} \longrightarrow \ldots \rightarrow X_{n}=Y .
$$

2. $Y \in C I R_{X}$.

Our task is to derive a contradiction; we will prove it by mathematical induction.

First, the initial assertion:

$$
\begin{aligned}
& \text { If } X=X_{0} \longrightarrow X_{1} \text { is a DIF, then } C I R_{X}=C I R_{X_{1}} \text { and } \\
& {[X]_{I A R}=\left[X_{1}\right]_{\text {IAR }} }
\end{aligned}
$$

To prove this assertion, note that $X_{1}$, receiving information from $X$, cannot be in $\operatorname{CIR}_{X}$. Hence, must be in its complement, that is,

$$
X_{1} \in[X]_{I A R}
$$

Since IAR an equivalence relation (FACT 2), we have

$$
\left[X_{1}\right]_{I A R}=[X]_{I A R} \text {. }
$$

By the fact that they are the complements, we have

$$
C I R_{X}=C I R_{X_{1}} .
$$

So the initial assertion is proved. 
Next, we consider the induction case. The arguments are essentially the same. By induction assumption, we have

$$
[X]_{I A R}=\left[X_{j}\right]_{I A R} \text { and } C I R_{X}=C I R_{X_{j}} .
$$

Next, consider the DIF, $X_{j} \longrightarrow X_{(j+1)}$.

Since $X_{(j+1)}$, receiving information from $X_{j}$, cannot be in $C I R_{X_{j}}$. By the FACT 2 again,

$$
X_{j+1} \in\left[X_{j}\right]_{I A R}=[X]_{I A R}
$$

By the FACTs that IAR is an equivalence and CIR is its complement, we have

$$
\begin{aligned}
& {[X]_{I A R}=\left[X_{j+1}\right]_{I A R},} \\
& C I R_{X}=C I R_{X_{j+1}} .
\end{aligned}
$$

This completes the induction proof. In particular

$$
X_{n}=Y \in[X]_{I A R} \text {. }
$$

This is contrary to one of the assumptions (Item 3.2). We have proved the strong Chinese wall security policy. That is, we have proved: SCWSP $\Longrightarrow$ ACWSP. The converse is obvious.

\section{DAC Models and Trojan Horses}

In this section, we take the DAC view on ACSWP model. If the agent $x$ explicitly denies the read access to $y$, then we say

The information flow from $X$ to $Y$ is denied, or equivalently,

No direct Information Flow (NIF) from $X$ to $Y$ is permitted, If the information flows between objects are regulated by NIF, we say $O$ is a flow oriented explicitly denied DAC model. NIF defines a Binary relation (NIFR) among all objects.

Definition : A Composite Information Flow from $X$ to $Y$ is called a malicious Trojan horse, if NIF from $X$ to $Y$ is the given requirement.

Trojan Horse Theorem. If NIFR is anti-reflexive, symmetric and anti-transitive, then the following three assertions are equivalent

Malicious Trojan horses from $X$ to $\mathrm{Y}$ may not occur

$\Longleftrightarrow$ DIF from $X$ to $Y$ may not occur

$\Longleftrightarrow(X, Y) \in \mathrm{NIFR}$.

NIFR is a DAC form of CIR, so this theorem follows immediate from Chinese wall theorem. 


\section{Some New Views of ACWSP}

The new observation on CIR gives a new insight to the CWSP-model. Here are the new views of theorems in [14] and [2].

THEOREM 1. Once a agent $S_{i}$ has accessed an object $O_{j}$, the only other objects $O_{k}$ accessible by $S_{i}$ is inside the allied dataset of $O_{j}$, which is the outside of $C I R_{O_{j}}$.

THEOREM 2. The minimum number of agents which allow every object to be accessed by at least one agent is $n$, where $n$ is the number of $E_{C I R}$-equivalence classes.

THEOREM 3. The flow of unsanitized information is confined to its allied dataset; sanitized information may, however, flow freely through the system.

\section{Conclusions}

The intuitive idea behind Brewer and Nash theory is useful for commercial security. By the new demands in eCommerce, as well as homeland security, we re-visited the BN-theory. In [19], we fuzzify the ACWSP model to make it more susceptible to uncertainty. In this paper, we refine ACWSP model by the new development of granular computing. The results are somewhat surprising, we can confine the malicious Trojan horses.

This paper realizes the BN's intend, however, it has more. From DAC's view, the results are just the beginning; it shows that information flows analysis on DAC is no longer a hopless subject. More analysis will be reported in near future.

\section{Appendix: Granular Computing for CIR}

Trojan horses in DAC model have been the weakest point of DAC model. Implicitly, BN's CWSP is designed to handle that. Unfortunately, their idea of using partition is a bit naive. This paper indicates that granulation seems a right notion. In next few sections, the new methodology will be explained.

\section{Equivalence Relations and Partitions}

A partition is a collection $\left\{N_{j} \mid j=1,2, \ldots\right\}$ of disjoint subsets whose union is $V$. This geometric notion defines naturally an algebraic notion, an equivalence relation, as follows:

$$
E=\cup_{j}\left\{(p, u) \mid p \text { and } u \text { are in } N_{j}\right\}
$$


This equivalence relation is reflexive, symmetric and transitive. $N_{j}$ is called an equivalence class in mathematics. It also has been referred to as an elementary set, a block, a granule [24],[10],[21]. Conversely, an equivalence relation defines a partition; we skip the details.

Algebraically, a natural generalization of an equivalence relation is a binary relation. Geometrically, a "common" generalization of a partition is a covering. Unfortunately, a covering is not the geometric equivalence of a binary relation. The equivalent one is a more elaborate notion; see next subsection.

\section{Binary Relations and Granulations}

Let $R \subseteq V \times U$ be a binary relation. We will re-express $R$ geometrically.

For each object $p \in V$, we associate a subset (binary set, neighborhood, or class) $N_{p} \subseteq U$ defined by

$$
N_{p}=\{u \in U \mid(p, u) \in R\}
$$

$N_{p}$ consists of all elements $v$ that are related to $p$ by the binary relation $R$. Geometrically, $N_{p}$ can be regarded as a neighborhood of $p$ in the following sense: the points in $N_{p}$ are "near to" or "related to" $p$ via $R$. We will use binary neighborhood to remind us that algebraically, it is defined by a binary relation, while geometrically, it consists of points in proximity. Intuitively, $N_{p}$ is the "nearest" neighborhood of $p$.

In BN-theory, CIR-classes are actually CIR-neighborhood. Just to be compatible with literature,we will use CIR-classes; readers should be cautioned that they are actually neighborhoods, not equivalence classes.

To help visualize the situation, we will use geometric language.

A binary granulation is an assignment that assigns to each object $p \in V$, a (possibly empty) subset $\boldsymbol{B}_{\boldsymbol{p}} \subseteq U$ :

$$
B: V \longrightarrow 2^{U} \text {. }
$$

We will refer to the collection, $\left\{B_{p} \mid p \in V\right\}$, a binary neighborhood system and each $B_{p}$ as a binary neighborhood of $p \in V$. They are called a basic neighborhood and a basic neighborhood system respectively in [16]. Though the subset $\boldsymbol{B}_{\boldsymbol{p}}$ sounds arbitrary, it can be regarded as a binary neighborhood $N_{p}$ of a binary relation, for all $p$. Formally, let us collect the comments into a

Proposition. Binary relation, binary granulation, and binary neighborhood system are equivalent.

In other words, given the map B (or the collection $\left\{B_{p} \subseteq U \mid p \in V\right\}$ ), there is a binary relation $R \subseteq V \times U$ such that $B_{p}=N_{p}$ and vice versa. So we will use the three terms interchangeably and denote all of them by $B$. The proof of the proposition is straight forward; we skip the details.

In application, a binary neighborhood at $p$

$$
B_{p}=\{v \mid(p, v) \in B\} .
$$

is often assigned a descriptive name, called elementary concept (or an attribute value in database community). The collection $C$ of all those elementary concepts is called the concept space (or attribute domain). Formally, we define 
A granular structure consists of 4-tuple

$$
(V, U, B, C)
$$

where $V$ is called the object space, $U$ is the data space ( $V$ and $U$ could be the same set), $B$ is a neighborhood system, and $C$ is the concept space which consists of all the names of the fundamental neighborhoods of $B$. If $B$ is a binary neighborhood system (binary relation), then the 4-tuple $(V, U, B, C)$ is called a binary granular structure.

\section{The Induced Partitions}

In $[17,18], T$. Y. Lin observed that the binary granulation relation,

$$
B: V \longrightarrow 2^{U}
$$

induced naturally a partition:

Consider the collection

$\left\{B^{-1}(w) \mid w \in 2^{U}\right\}$, called inverse image of $w$ under $B$.

It is easy to verify that the collection forms a partition on $V$. We call it the induced partition of $B$, and denoted by $E_{B}$.

The equivalence class, $[p]_{E_{B}}=B^{-} 1\left(B_{p}\right)$, or simply write as $[p]$, is called the center or core of $B_{p}$.

Proposition 1. The center $[p]$ consists of all those points that have the same binary neighborhood $B_{\boldsymbol{p}}$ ("same" in the sense of set theory).

Proposition 2. If $B \subseteq V \times V$ is a symmetric binary relation, and $E_{B}$ is its induced equivalence relation, then each $B$-binary neighborhood is a union of $E_{B}$-equivalence classes.

Proof: Let $B_{p}$ be the binary neighborhood of $p$. Let $x \in B_{p}$, and assume $x$ and $y$ are $E_{B}$-equivalence. By definition of equivalence in $E_{B}$, they have the same neighborhood, $B_{x}=B_{y}$. By the symmetry of $B, x \in B_{p}$ implies $p \in B_{x}=B_{y}$, and hence $y \in B_{p}$. In other words, both $x$ and $y$ are in $B_{p}$. Since $y$ is arbitrary, this proves $[x] \subseteq B_{p}$, that is $B_{p}$ contains the equivalence class $[x]$ of its member $x$. QED

This proposition is one of the main technical results that have a strong impact on Chinese Walls. The condition of symmetry in this proposition is needed: In the oringal version examples were given; for space consideration; we skip the examples.

\section{The Complement of a Binary Relation}

First some definitions

A symmetric binary relation $\mathrm{B}$ is a binary relation such that for every $(u, v) \in B$ implies $(v, u) \in B$.

A binary relation $\mathrm{B}$ is anti-reflexive, if $B$ is non-empty and no pair $(\nu, v)$ is in $B$. 
That is, $B \cap \Delta=\emptyset$, where $\Delta=\{(v, v) \mid v \in V\}$ is the diagonal set.

A binary relation $\mathrm{B}$ is anti-transitive, if $B$ is non-empty and if $(u, v)$ belongs to $B$ implies that for all $w$ either $(u, w)$ or $(w, v)$ belongs to $B$.

$B^{\prime}=V \times V \sim B$, which is the complement set of $\mathrm{B}$, defines the complement binary relation $(\mathrm{CBR})$.

Proposition 3. If $B$ is symmetric, anti-reflexive and anti-symmetric, then the complement binary relation $B^{\prime}$ is an equivalence relation.

Proof: $B$ is anti-reflexive, so the diagonal set is contained in the complement, that is, $\boldsymbol{B}^{\prime}$ is reflexive. We will prove by indirect method: Assume to the contrary that $(\boldsymbol{u}, \boldsymbol{w})$ and $(w, v)$ are in $B^{\prime}$ and its composition $(u, v)$ were not (that is, $(u, v) \in B$ ). By anti-transitivity of $B$, we should either $(u, w)$ and $(w, v)$ belong to $B$; a contradiction. So the assumption $(u, v) \in B$ is incorrect; This leads to $\left.(u, v) \in B^{\prime}\right)$; QED

Corollary 4. If $B$ is symmetric, anti-reflexive and anti-transitive, then $B^{\prime}$ is the induced equivalence relation $E_{B}$.

Proof: Let $v \in V$ and $B_{v}$ be its binary neighborhood. First we want to prove $\boldsymbol{B}^{\prime} \subseteq E_{\boldsymbol{B}}$ : That means we need to show that for any $u$, which is $\boldsymbol{B}^{\prime}$-equivalent to $v$, is $E_{\boldsymbol{B}}$-equivalent to $\boldsymbol{v}$, that is, $\boldsymbol{B}_{\boldsymbol{v}}=\boldsymbol{B}_{\boldsymbol{u}}$.

First, we will show $B_{v} \subseteq B_{u}$ : Note that $(u, v) \in B^{\prime}$, by assumption. Let $p$ be a point in the $B_{v}$, that is, $(v, p) \in B$. Then, by anti-transitive and symmetry, $(u, p)$ belong to $B$ (otherwise, $(u, v) \in B$, which is absurd). That is, $p$ is in the neighborhood of $u$. So we have proved that $B_{v} \subseteq B_{u}$.

Let $q \in B_{u}$ plays the role as $p$, by a similar arguments, We can show that $B_{u} \subseteq B_{v}$. So we have proved $\boldsymbol{u}$ and $\boldsymbol{v}$ have the same neighborhood, that is, $B_{u}=B_{v}$. This conclude the proof: $B^{\prime} \subseteq E_{B}$.

Second, we will prove the reverse inclusion $E_{B} \subseteq B^{\prime}$ : Note that $(u, v) \in E_{B}$ implies $\boldsymbol{B}_{\boldsymbol{u}}=\boldsymbol{B}_{\boldsymbol{v}}$. Let $\boldsymbol{p}$ be a point in $\boldsymbol{B}_{\boldsymbol{u}}=\boldsymbol{B}_{\boldsymbol{v}}$, that is, both $(\boldsymbol{u}, \boldsymbol{p})$ and $(\boldsymbol{p}, \boldsymbol{v})$ belong to $B$. By anti-transitivity and symmetric, $(u, v)$ belongs to $B^{\prime}$. This proves the Corollary. QED

\section{References}

[1] Bell, D. 1987. Secure computer systems: A network interpretation. In Proceedings on 3rd Annual Computer Security Application Conference. 32-39.

[2] David D. C. Brewer and Michael J. Nash: "The Chinese Wall Security Policy" IEEE Symposium on Security and Privacy, Oakland, May, 1988, pp 206-214.

[3] Richard A. Brualdi, Introductory Combinatorics, Prentice Hall, 1992.

[4] W. Chu and Q. Chen Neighborhood and associative query answering, Journal of Intelligent Information Systems, 1, 355-382, 1992.

[5] S. A. Demurjian and S. A. Hsiao "The Multimodel and Multilingual Database Systems-A Paradigm for the Studying of Database Systems, "IEEE Transaction on Software Engineering, 14, 8, (August 1988) 
[6] Denning, D. E. 1976. A lattice model of secure information flow. Commun. ACM 19,2, 236-243.

[7] Hsiao, D.K., and Harary, F., "A Formal System for Information Retrieval From Files," Communications of the ACM, 13, 2(February 1970). Corrigenda CACM 13,3 (March, 1970)

[8] Wong, E., and Chiang, T. C., "Canonical Structure in Attribute-Based File Organization," Communications of the ACM, Vol. 14, No. 9, September 1971.

[9] C. E. Landhehr, and C. L. Heitmeyer:Military Message Systems:Requirements and Security Model, NRL Memorandom Report 4925, Computer Science and Systemss Branch, Naval research Laboratory.

[10] T. T. Lee, "Algebraic Theory of Relational Databases," The Bell System Technical Journal Vol 62, No 10, December, 1983, pp.3159-3204

[11] T. Y. Lin, Neighborhood Systems and Relational Database. In: Proceedings of 1988 ACM Sixteen Annual Computer Science Conference, February 23-25, 1988, 725

[12] "A Generalized Information Flow Model and Role of System Security Officer", Database Security: Status and Prospects II, IFIP-Transaction, edited by C. E. Landwehr, North Holland, 1989, pp. 85-103.

[13] T. Y. Lin, Neighborhood Systems and Approximation in Database and Knowledge Base Systems, Proceedings of the Fourth International Symposium on Methodologies of Intelligent Systems , Poster Session, October 12- 15, pp. 7586, 1989.

[14] T. Y. Lin, "Chinese Wall Security Policy-An Aggressive Model”, Proceedings of the Fifth Aerospace Computer Security Application Conference, December 4-8, 1989, pp. 286-293.

[15] "Attribute Based Data Model and Polyinstantiation," Education and Society, IFIP-Transaction, ed. Aiken, 12th Computer World Congress, September 7-11, 1992, pp.472-478.

[16] T. Y. Lin, "Neighborhood Systems -A Qualitative Theory for Fuzzy and Rough Sets," Advances in Machine Intelligence and Soft Computing, Volume IV. Ed. Paul Wang, 1997, Duke University, North Carolina, 132-155. ISBN:0-9643454-3-3

[17] T. Y. Lin "Granular Computing on Binary Relations I: Data Mining and Neighborhood Systems." In: Rough Sets In Knowledge Discovery, A. Skoworn and L. Polkowski (eds), Physica-Verlag, 1998, 107-121

[18] T. Y. Lin "Granular Computing on Binary Relations II: Rough Set Representations and Belief Functions." In: Rough Sets In Knowledge Discovery, A. Skoworn and L. Polkowski (eds), Physica - Verlag, 1998, 121-140.

[19] T. Y. Lin "Chinese Wall Security Model and Conflict Analysis," the 24th IEEE Computer Society International Computer Software and Applications Conference (Compsac2000) Taipei, Taiwan, Oct 25-27, 2000

[20] T. Y. Lin "Feature Completion," Communication of IICM (Institute of Information and Computing Machinery, Taiwan) Vol 5, No. 2, May 2002, pp. 57-62. This is the proceeding for the workshop "Toward the Foundation on Data Mining" in PAKDD2002, May 6, 2002.

[21] T. Y. Lin "A Theory of Derived Attributes and Attribute Completion," Proceedings of IEEE International Conference on Data Mining, Maebashi, Japan, Dec 9-12, 2002.

[22] A, Motro: "Supportin Gaol Queries", in :Proceeding of the First Internatonal Conference on Expert Database Systems, L. Kerschber (eds)m April 1-4, 1986, pp. 85-96. 
[23] S. Osborn, R. Sanghu and Q. Munawer, "Configuring RoleBased Access Control to Enforce Mandatory and Discretionary Access Control Policies," ACM Transaction on Information and Systems Security, Vol 3, No 2, May 2002, Pages 85-106.

[24] Z. Pawlak, Rough sets. International Journal of Information and Computer Science 11, 1982, pp. 341-356.

[25] Z. Pawlak, "On Conflicts," Int J. of Man-Machine Studies, 21 pp. 127- 134, 1984

[26] Z. Pawlak, Analysis of Conflicts, Joint Conference of Information Science, Research Triangle Park, North Carolina, March 1-5, 1997, 350- 352.

[27] Polkowski, L., Skowron, A., and Zytkow, J., (1995), “Tolerance based rough sets.” In: T.Y. Lin and A. Wildberger (eds.), Soft Computing: Rough Sets, Fuzzy Logic Neural Networks, Uncertainty Management, Knowledge Discovery, Simulation Councils, Inc. San Diego CA, 55-58.

[28] Sandhu, R. S. Latticebased enforcement of Chinese Walls. Computer \& Security 11, 1992, 753-763.

[29] Sandhu, R. S. 1993. Latticebased access control models. IEEE Computer 26, 11, 9-19.

[30] Sandhu,R.S.,Coyne,E.J.,Feinstein,H.L., and Youman, C. E. 1996. Rolebased access control models. IEEE Computer 29, 2 (Feb.), 38 - 47.

[31] Sandhu,R.AND Munawer, Q. 1998. How to do discretionary access control using roles. In Proceedings of the Third ACM Workshop on RoleBased Access Control (RBAC '98, Fairfax, VA, Oct. 22-23), C. Youman and T. Jaeger, Chairs. ACM Press, New York, NY, 47-54.

[32] W. Sierpinski and C. C, Kreiger, General Topology, University Toronto press, 1952

[33] T.C. Ting, "A User-Role Based Data Security Approach", in Database Security: Status and Prospects, C. Landwehr (ed.), North-Holland, 1988.

[34] Demurjian, S., and Ting, T.C., "Towards a Definitive Paradigm for Security in Object-Oriented Systems and Applications," J. of Computer Security, Vol. 5, No. 4, 1997.

[35] Liebrand, M., Ellis, H., Phillips, C., Demurjian, S., Ting, T.C., and Ellis, J., "Role Delegation for a Resource-Based Security Model," Data and Applications Security: Developments and Directions II, E. Gudes and S. Shenoi (eds.), Kluwer, 2003.

[36] Phillips, C., Demurjian, S., and Ting, T.C., "Towards Information Assurance in Dynamic Coalitions," Proc. of 2002 IEEE Info. Assurance Workshop, West Point, NY, June 2002.

[37] L.A. Zadeh, Fuzzy sets and information granularity, in: M. Gupta, R. Ragade, and R. Yager (Eds.), Advances in Fuzzy Set Theory and Applications, NorthHolland, Amsterdam, 3-18, 1979.

[38] L. Zadeh, "Some Reflections on Information Granulation and its Centrality in Granular Computing, Computing with Words, the Computational Theory of Perceptions and Precisiated Natural Language." In: T. Y. Lin, Y.Y. Yao, L. Zadeh (eds), Data Mining, Rough Sets, and Granualr Computing T. Y. Lin, Y.Y. Yao, L. Zadeh (eds) 